Insciences J. 2011, 1 (1), 18-29; doi:10.5640/insc.010118

Full Research Paper

\title{
Investigation of Metallic Elliptical Nano-Pinholes Structure-Based Plasmonic Lenses: From Design to Testing
}

\author{
Yiwei Zhang, Yongqi Fu* and Xiuli Zhou
}

School of Physical Electronics, University of Electronic Science and Technology of China, Chengdu 610054, Sichuan Province, P.R. China

* Author to whom correspondence should be addressed; E-Mail: yqfu@uestc.edu.cn

Received: 12 August 2010 / in revised form: 12 October 2010 / Accepted: 26 November 2010 / Published: 02 January 2011

\begin{abstract}
A novel metallic elliptical nano-pinholes-based plasmonic lens was put forth for the purpose of superfocusing. Systematic investigation of the lens from design to fabrication and characterization were described in this paper. Surface plasmon polaritons (SPPs) enhanced transmission is theoretically analyzed using finite-difference time-domain (FDTD) algorithm-based computational calculation. Then the designed pattern was fabricated using focused ion beam (FIB) directly milling technique. Focusing of the lens is observed from the results of both theoretical calculation and experimental characterization using near-field scanning optical microscope (NSOM). The experimental results are in agreement with that of the theoretical calculation. Our results demonstrated that the lens can realize subwavelength focusing with enhanced transmission, elongated focal length, and depth of focus.
\end{abstract}

Keywords: plasmonic lenses; FIB; elliptical nano-pinhole; NSOM; FDTD.

\section{Introduction:}


With rapid development of optical beaming in applications of biosensing, imaging, and data storage, tightly focusing in nanoscale confinement of surface plasmon polaritons (SPPs) has been studied actively. Many types of plasmonic lenses appeared subsequently such as metallic cone-shaped waveguide [1, 2], width modulation-based lenses [3-5], elliptical nanopinhole lens [6, 7], single circular ring-based lens [8-11], circular pinholes lens [12], nanowaveguide focusing [13], radial polarization-based circular grating lens [14, 15], photon sieve [16], and polarization-based elliptical nanohole arrays [17, 18]. All of the reported structures have functions and advantages of focusing surface plasmons, superfocusing within the range of micron-scale along propagation direction and spatial resolution beyond diffraction limit, and extraordinary transmission. Ag thin film-based elliptical nano-pinhole structured plasmonic lens was theoretically studied by means of computational numerical calculation on the basis of finite-difference time-domain (FDTD) algorithm in Ref. [6] before.

In this paper, we gave a systematic investigation of the lenses from designing to experimental issues. Transmission property and focusing performance were addressed by means of FDTD-based calculation firstly. Then further experimental study of the plasmonic lens was carried out through nanofabrication, and optical characterization. Focused ion beam direct milling technique with one-step etching process was employed to manufacture the lens. A near-field scanning optical microscope (NSOM) has been adopted for the purpose of evaluating optical performance of the lens. Our experimental results demonstrated that the lenses can realize subwavelength focusing, elongated focal length, and extended depth of focus.

\section{Lens structuring and experimental setup:}

The plasmonic lens is composed of elliptical pinholes designed with different sizes distributed in different rings with variant periods, as shown in Fig. 1 (a). Long-axis of the ellipse is defined as $a_{n}=3 \omega_{n}$, whereas $\omega_{n}$ is the width of the corresponding ring width, and $n$ is the number of the rings. A metallic monolayer thin film of Au coated on the glass substrate is perforated by the pinholes. There are total 8 rings on the metal film. The pinholes are completely penetrated through the Au film. The number of pinholes from inner to outer rings is $8,20,36,55,70,96,107$, and 140 , respectively. Outer diameter of the ring is $12.05 \mu \mathrm{m}$. Radius of the rings can be calculated by the formula $r_{n}^{2}=2 n f \lambda+n^{2} \lambda^{2}$, where $f$ is the focal length for working wavelength of $\lambda=633 \mathrm{~nm}$. The formula was deduced in terms of optical path difference $\lambda$ by the relationship of $f^{2}+r_{\mathrm{n}}{ }^{2}=(f+n \lambda)^{2}$, where $n$ is integer number of rings, $f$ is focal length, and $r_{\mathrm{n}}$ is $\mathrm{n}^{\text {th }}$ radius of the rings. Advantage of the elliptical pinholes in comparison to circular grating metallic structure is that the pinhole lens has elongated focal length and extended depth of focus $(D O F)$ [6]. The focal region is formed by interference between the cavity mode-coupled SPPs wave and diffraction wavelets passing through the rings designed as the metallic dielectric-air (MDA) waveguides formed by the Au films as well as their clad dielectric layers. For the plasmonic lenses, the energy flow will be guided toward the focal points of the lenses. Each pinhole here acts as a small lasing cavity with 
Fabry_Pérot (FP) resonance_which contributes on the final focusing with ultra-long DOF. The final intensity at the focal point is synthesized by iteration of each zone focusing and interference each other, and can be expressed as

$$
I=\alpha \sum_{i=1}^{N} C I_{i 0} I_{S P} \frac{4 r_{i}}{\lambda_{S P}} e^{-\left(r_{i} / l_{S P}\right)}
$$

where $I_{i 0}$ is the intensity of diffractive wavelet at $\mathrm{i}^{\text {th }}$ zone, $I_{\mathrm{SP}}$ is the intensity of the SPPs wave passing through the $\mathrm{i}^{\text {th }}$ slit, $r_{\mathrm{i}}$ is the radius of each zone, $i$ is the number of the zones, $l_{\mathrm{SP}}$ is the propagation length for the SPPs wave, $\alpha$ is interference factor, and $C$ is the coupling efficiency of the slits. $C$ is a complicated function of the slit geometry and will likely have a different functional form when the slit width is much larger or much smaller than the incident wavelength.

The micrograph of scanning electron microscope (SEM) of the FIB fabricated lens is shown in Fig. 1 (b). Vertical sidewall of the holes will be changed like cone/V-shape (slanted sidewall) due to redeposition of the FIB process. Our previous relevant theoretical calculation results showed that the cone shape plays a positive contribution for enhancement of transmission intensity [19].

Figure 1. (a) Schematic diagram of the pinholes-based plasmonic lens with focal length $f$. The pinholes are uniformly distributed along the zones. The lens is under illumination with linear polarized plane wave and $532 \mathrm{~nm}$ incident wavelength. (b) SEM image of FIB fabrication result.

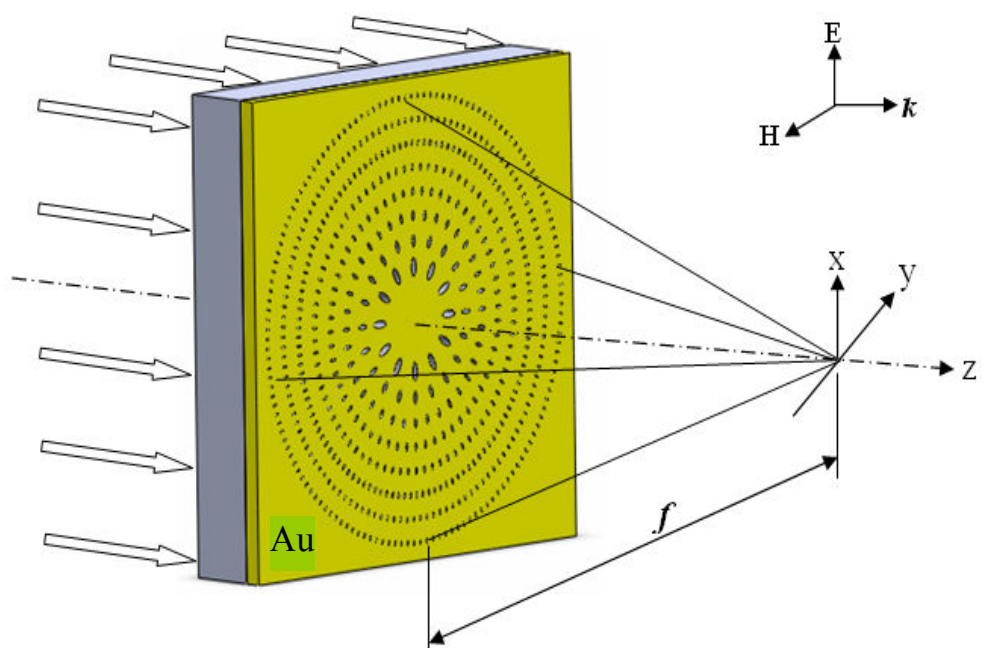

(a) 


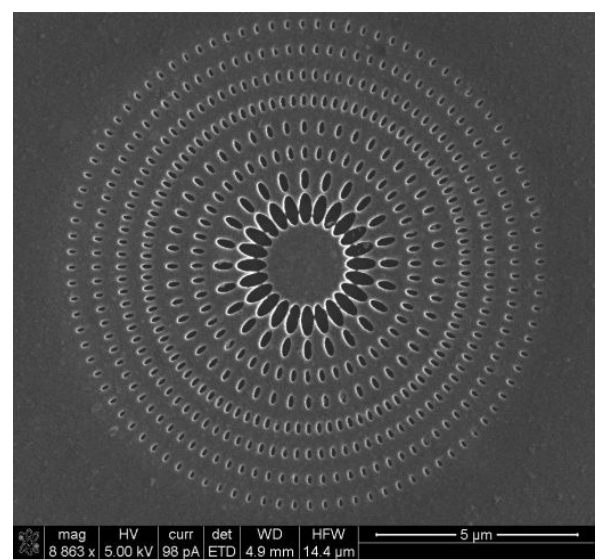

(b)

The lengths in short-axis of the pinholes are ranging from $55 \mathrm{~nm}$ (outmost) to $194 \mathrm{~nm}$ (inner). The ratio between the long- and short-axis of all the pinholes was designed to be constant in value of 0.2 . However, it comes out to be 0.4 due to the fabrication error of inherent FIB line broadening effect. As can be seen from Fig. 2, when the ratio of short-axis / long-axis changes from 0.1 to 0.8 , the transmission variation is near $20 \%$. For the elliptical pinholes, the maximum transmission occurred when the light was polarized perpendicular to the long-axis of the ellipse; as the ellipse is rotated, the polarization followed the ellipse orientation. The ellipses had a constant maximum intensity for different basis orientations as long as the ratio is fixed. The maximum polarization of transmission can follow the orientation of the ellipse, depending upon the shape of the ellipse chosen. With the ellipse, the combined coupling to the $(1,0)$ and $(0,1)$ modes resonances is unchanged, whereas the coupling changes as the holes are varied. The ellipse alone also polarizes the light transmission because of the polarization-dependent transmission of the lowest-order evanescent mode of the hole. It has been shown that the lowest-order mode plays a dominant role in the transmission of the nanoholes. For more information, readers can see Ref. [20, 21].

To obtain the E-field distribution across horizontal planes of different heights slightly above the structure, we used NSOM (MutiView2000, from Nanonics Inc.) working in collection mode. The lens is excited by YAG laser beam delivered from far-field eyepiece and adaptor into objective lens $(\times 20)$, and the transmitted light is detected through probe in nearfield. The fiber probe with $150 \mathrm{~nm}$ aperture was adopted for the optical characterization. Theoretically, the smaller aperture of the probe, the higher scanning resolution of the NSOM will be. However, the probe with small aperture (e.g., $50 \mathrm{~nm}$ aperture) will affect its photon collection and causes signal intensity is too low to image. For our probed plasmonic lens, considering FWHM $>>50 \mathrm{~nm}$ for which the influence of probe aperture on scanning resolution is not a crucial issue in this case, it is better to use the probes with larger aperture so as to ensure the collected signal intensity. Light source is a $532 \mathrm{~nm}$ wavelength laser since the working wavelength of the lenses was designed according to this value. The light is focused to a spot at the surface of the lens and is collected by the probe directly, so it is strong enough to be detected along propagation direction at surface of the exit plane in free space. We 
manually set height of the probe and scan the plane in current height. Here it is worthy to mention that for the NSOM measurement, actually, the lateral component of $\left|E_{x}\right|^{2}$ or $\left|E_{y}\right|^{2}$ plays an important role for the lens scanning under the illumination with linear polarization (see the relevant statement described in Ref. [22]). This is the reason we used the component $\left|\mathrm{E}_{\mathrm{x}}\right|^{2}$ as the simulation intensity in comparison to the NSOM measurement results here.

Figure 2. Normalized transmission of the nano-pinhole structured plasmonic lens vs. propagation distance $\mathrm{Z}$ for different ratios of short-axis to long-axis $\delta=0.1 \sim 0.8$ of the elliptical pinholes.

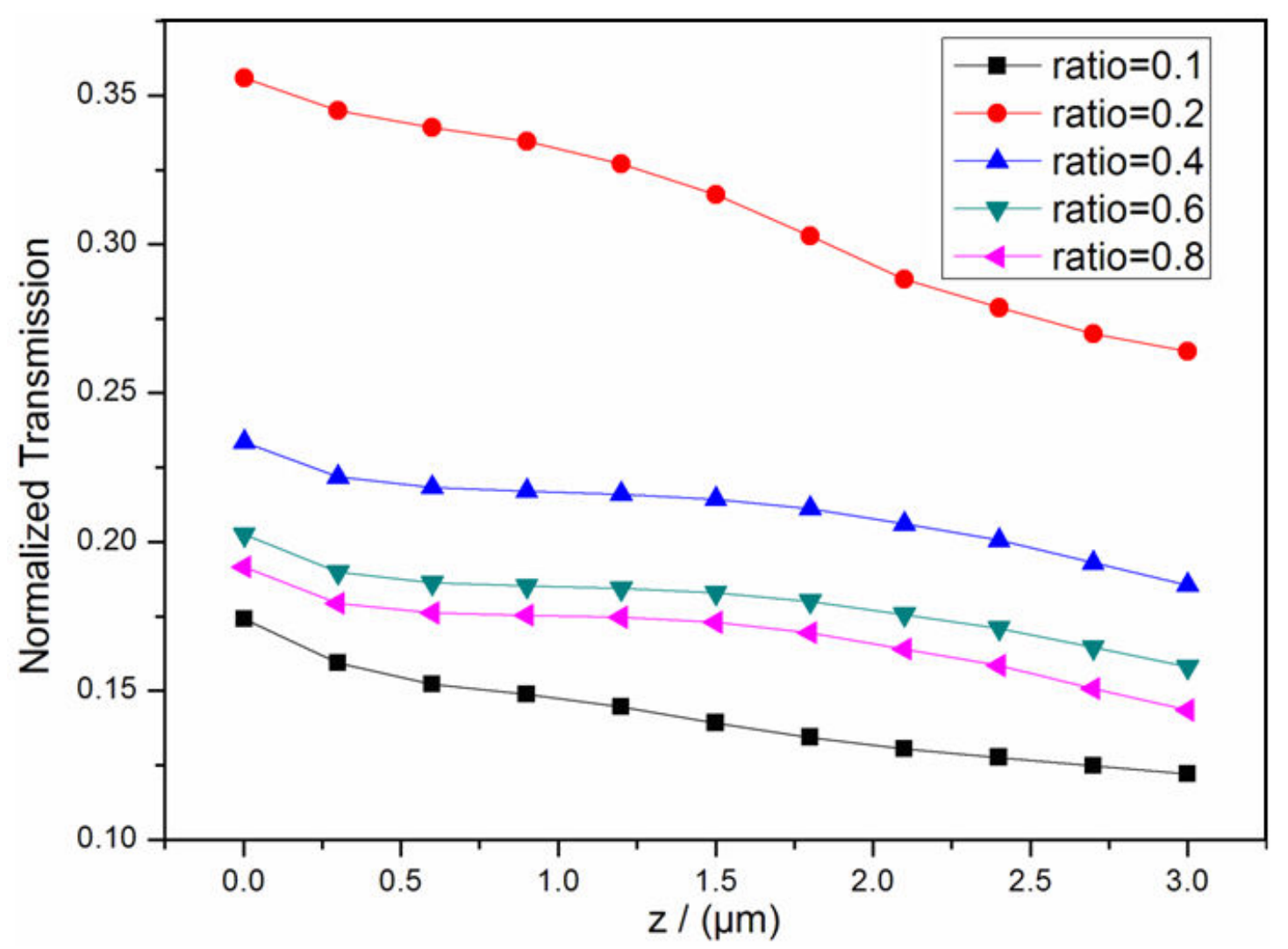


Figure 3. Calculated transmission spectra of the nano-pinhole structured plasmonic lens for different ratios of short-axis to long-axis $\delta=0.1 \sim 0.8$ of the elliptical pinholes.

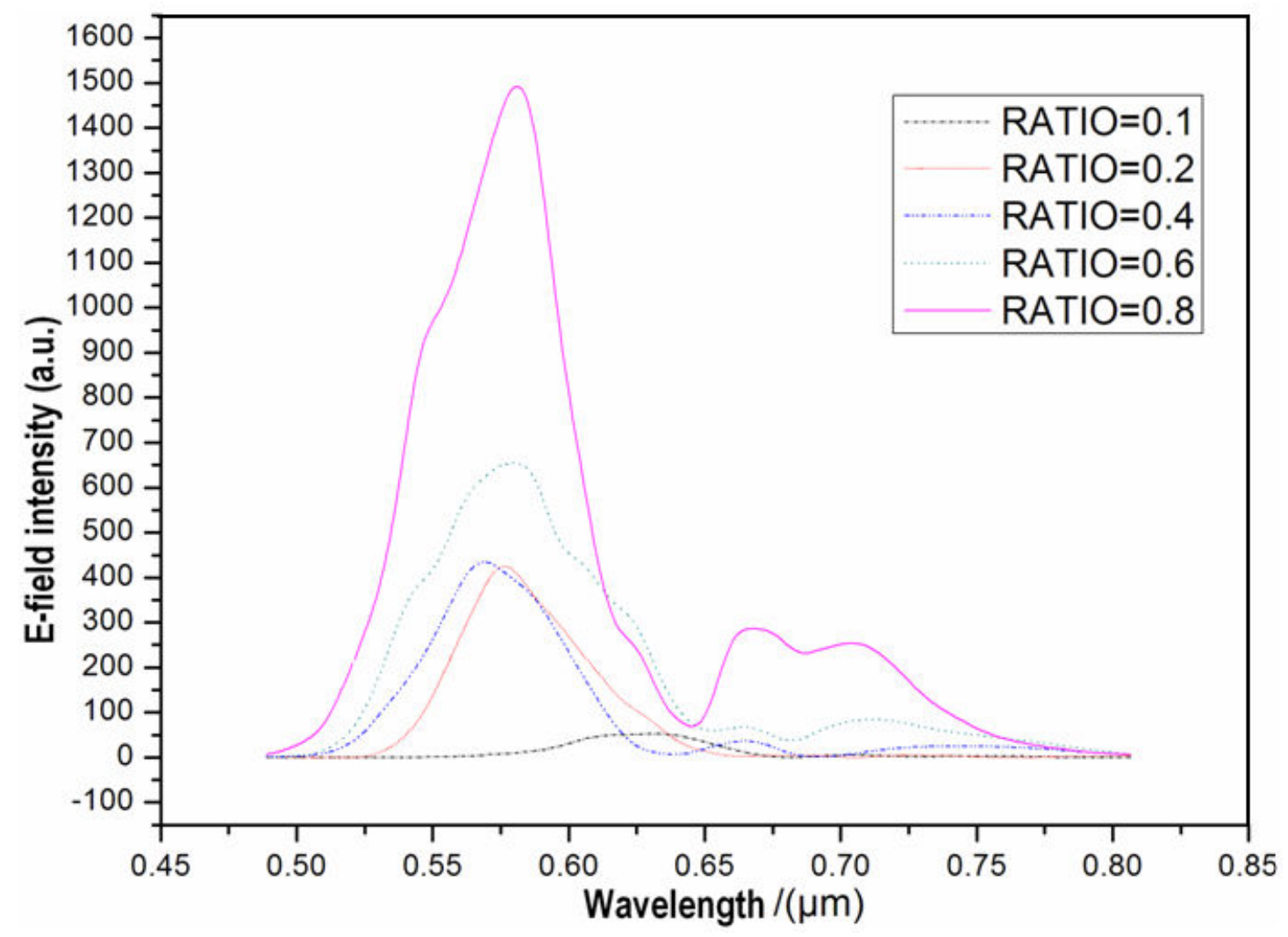

\section{Results and Discussion:}

Polarization issue is a crucial factor for transmission of the elliptical nano-pinholes-based structures [23]. Considering this, firstly, we addressed and theoretically analyzed the elliptical nano-pinholes-based lens with different ratios of short-axis to long-axis $\delta=0.1,0.2,0.4,0.6$, and 0.8 , respectively. Figure 2 is our calculated transmission vs. propagation distance $\mathrm{Z}$ for the different ratios $\delta$. It can be seen that the best transmission can be obtained for the ratio of $\delta=0.2$ due to the influence of the linear polarization. The calculated spectra in Fig. 3 show that position of resonant peaks is blue-shift firstly with increasing of $\delta$, and then red shifting for the case of $\delta>0.4$. It may attribute to the balance of polarization of the elliptical nano-pinholes and FP cavity-based SPPs propagating through the holes. Intensity distribution of $\left|E_{x}\right|^{2}$ at $x-y$ plane at $\mathrm{Z}=20 \mathrm{~nm}$ and $2.0 \mu \mathrm{m}$, respectively. Both theoretical computational calculation and NSOM probing results were shown as Figs. 4 (a)-(d)). Figure 4 demonstrates an agreement between theoretical calculation and experimental results at $Z=20 \mathrm{~nm}$ which is quite near the exit plane of the lens. In this near-field region, the interference-generated focusing spot has not been formed yet. However, both theoretical and experimental results show that the intensive focusing spot is formed at $Z=2.0 \mu \mathrm{m}$, as shown in Fig. 4 (c) and (d). Slight offset of the central focal spot (denoted as " $A$ " in the figure) is observed from Fig. 4 (d) due to misalignment of the light source during probing. In Fig. 4 (d), the measured minimum feature size of the localized field is $\mathrm{FWHM}=0.661 \mu \mathrm{m}$ at $\mathrm{Z}=2 \mu \mathrm{m}$. In contrast, the simulated spot size 
shown in Fig. 4 (c) is FWHM=0.276 $\mu \mathrm{m}$ at the same propagation distance. The difference between the measurement and simulation originates from fabrication and NSOM probing errors. The former causes broadening of the holes size due to inherent FIB broadening effect [24], especially for the inner holes; and the latter is mainly influenced by photons interaction between Al coating of the fiber probe and $\mathrm{Au}$ film of the lens while the probe scans the sample surface.

Figure 4. E-field intensity distribution of $\left|E_{x}\right|^{2}$ at $x-y$ plane at propagation distance of (a) and (b) $\mathrm{Z}=20 \mathrm{~nm}$; (c) and (d) $\mathrm{Z}=2 \mu \mathrm{m}$. (a) and (c) are calculation results; (b) and (d) are corresponding NSOM measured results.

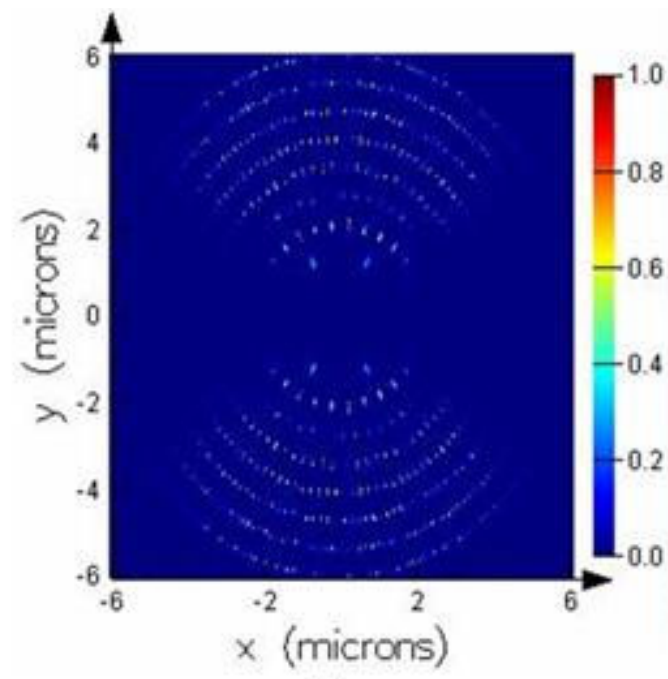

(a)

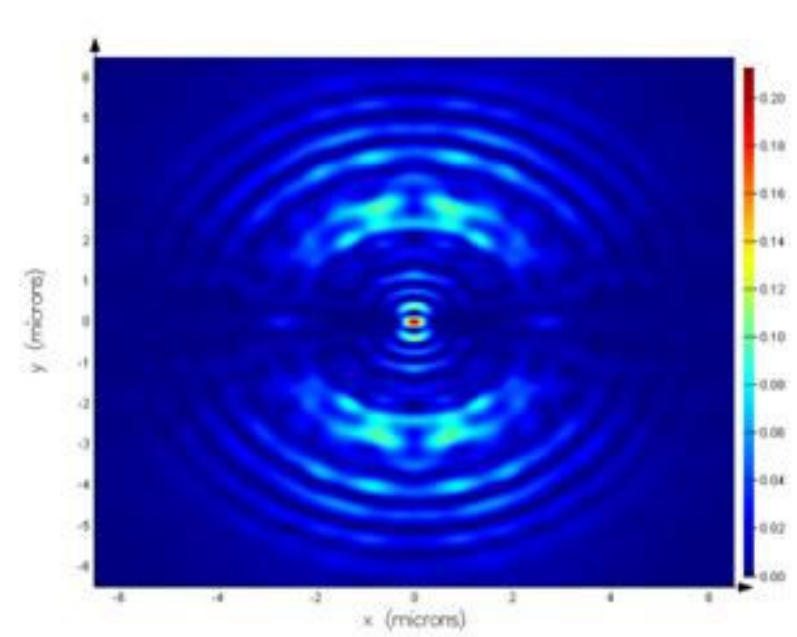

(c)

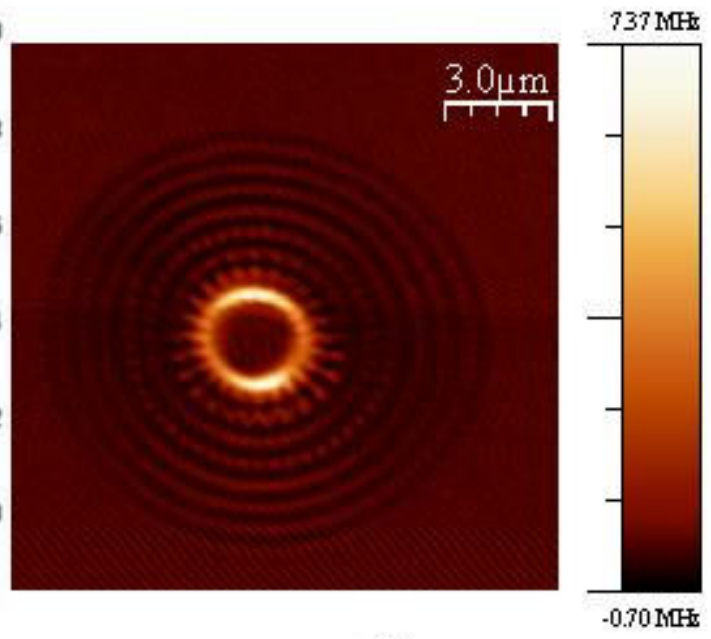

(b)

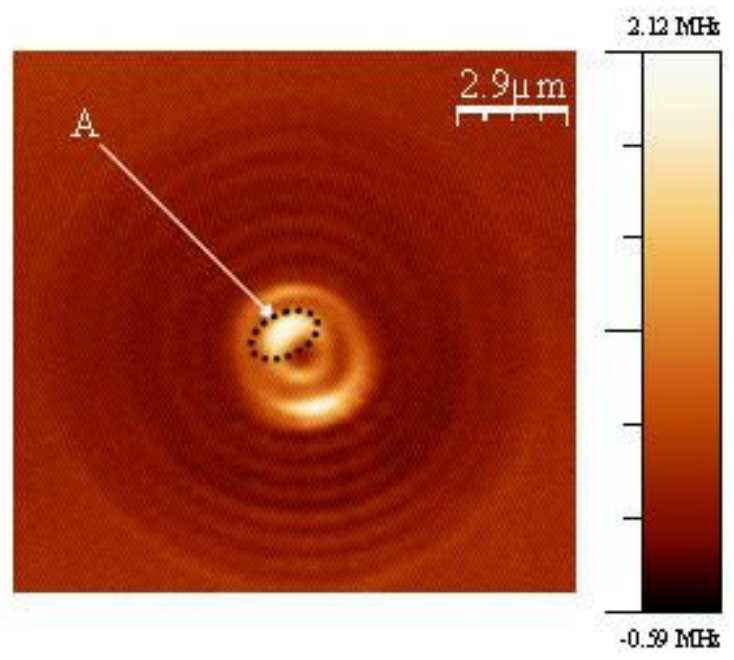

(d) 
Our experiments mainly demonstrated the focusing phenomenon of the novel lens. Before the NSOM probing, we adjusted intensity of the spot size of incident light to be as low as possible and then shifted optical axis of the illumination beam to center of the sample. Some errors were possibly involved in this step because the centering process was judged by the operator's nuked eyes only. The illumination region is slight larger than the size of the lens. There was a significant enhancement of intensity of light when we moved the spot from nonstructured Au film area to the lens. As scanning, the probe scans the lens in raster scan mode. To further illustrate the focusing, we gave the relationship between focusing spot and Z: both calculated and measured full-width and half-maximum (FWHM) as function of propagation distance Z, as shown in Fig. 5. It can be seen that calculated FWHM is $0.218 \mu \mathrm{m}$ (i.e., $0.41 \lambda$ ) and $0.276 \mu \mathrm{m}$ (i.e., $0.52 \lambda$ ) at propagation distance of $\mathrm{Z}=1$ and $2 \mu \mathrm{m}$, respectively. In contrast, Abbe diffraction limit for a conventional lens with the same outer diameter $(12.05 \mu \mathrm{m})$ and focal lens $(f=2 \mu \mathrm{m})$ is $2.458 \mu \mathrm{m}$. For $Z<1 \mu \mathrm{m}$, there is no focusing spot formed in this region. We measured the spot size along two perpendicular X-and Y-axes. And it shows obvious focusing effect in both the two directions along propagation distance. Even at the propagation distance as long as $Z=5 \mu \mathrm{m}$, the apparent focusing spot still can be seen, as shown in Fig. 6 (a) and (b). The measured focused region starts to become divergence for $Z>9 \mu \mathrm{m}$ though the calculated value shows that the beam is still focused with low intensity at $Z=9 \sim 10 \mu \mathrm{m}$, because of the inherent fabrication and measurement errors. Measured DOF is around $8 \mu \mathrm{m}$. The lateral extension of the focus is $\sim 120 \mathrm{~nm}$ within the DOF. It is in accordance with our previous theoretical calculation and analyses reported in Ref. [6].

Figure 5. Analyses of focusing performance: calculated and measured FWHM vs. propagation distance $\mathrm{Z}$.

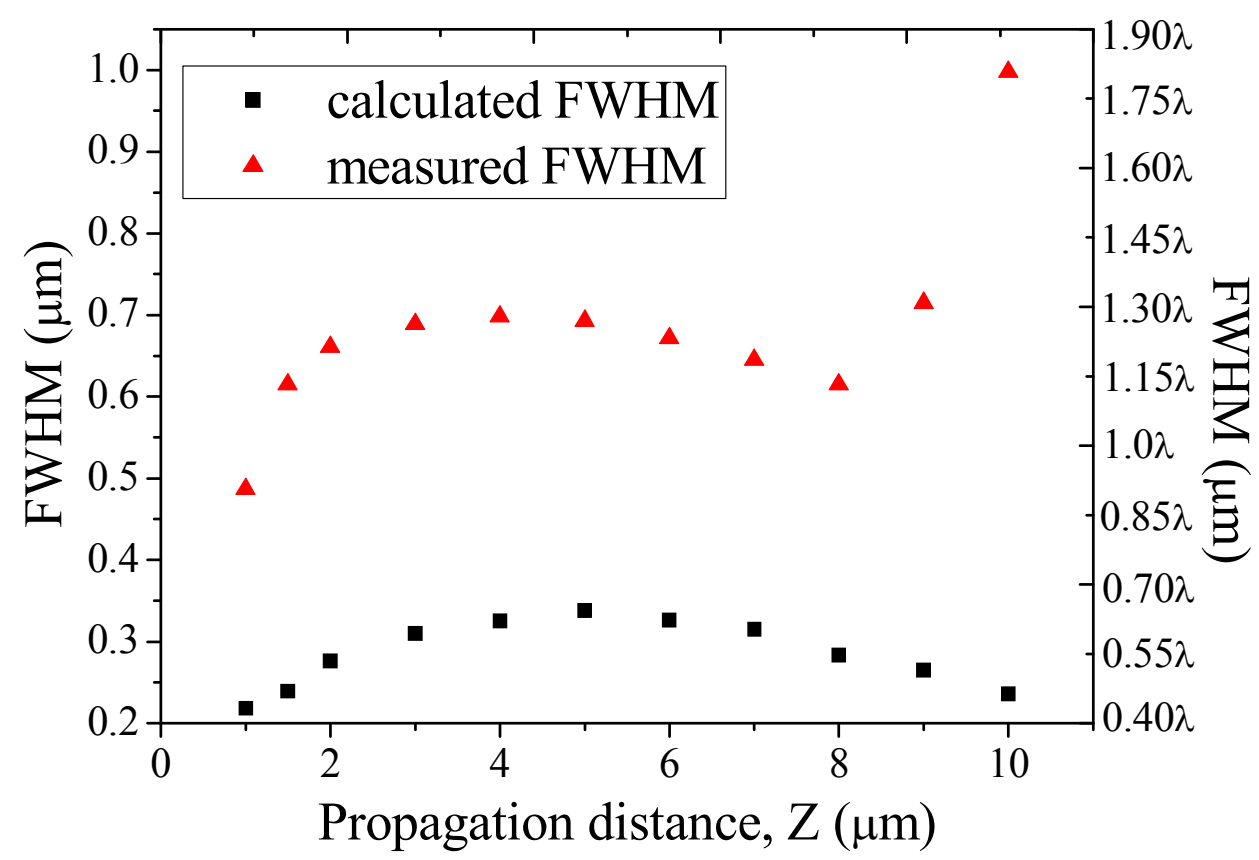


Figure 6. NSOM results regarding focusing performance with sample scanning along propagation direction at distance $Z=5 \mu \mathrm{m}$. NSOM image of (a) $2 \mathrm{D}$ and (b) $3 \mathrm{D}$.

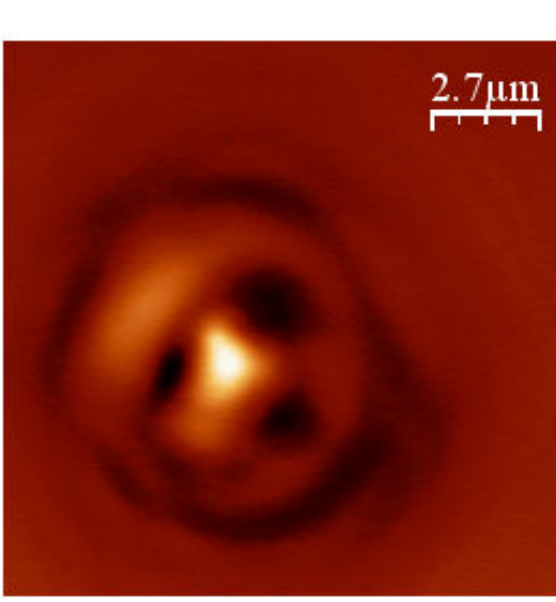

(a)

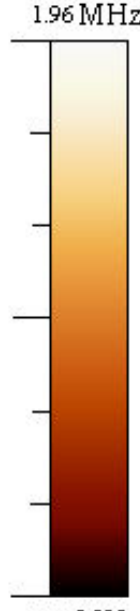

$-0.38 \mathrm{MHz}$

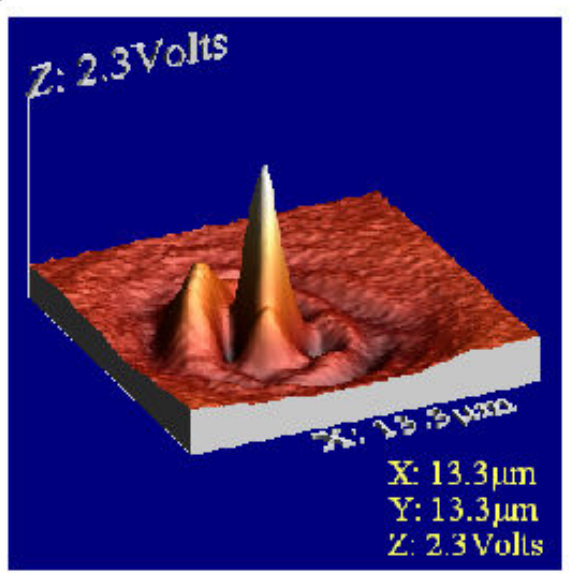

(b)

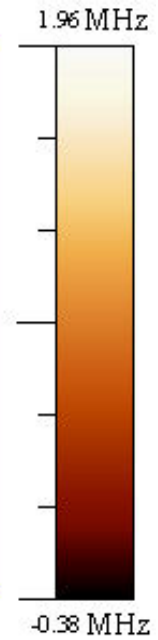

$-0.38 \mathrm{MHz}$

According to Ref. [14], there is a pattern with two separate sidelobes besides the central elliptical main lobe across the annular slits-based plasmonic lens under illumination with linear polarization (it also can be seen from our lens, as shown in Fig. 4 (c)). It can be improved to be one central circular focusing spot surrounded by the sidelobes with suppressed low intensity by means of using the light source with radial polarization as the illumination [15]. However, for our elliptical nano-pinholes-based lens here, the corresponding calculation result shows that the circular shaped focusing spot cannot be formed under the illumination with radial polarization, as shown in Fig. 7 (a). It can be explained that direction of the radial polarization is coincident with orientation of the elliptical pinholes in long-axis. Hence there is no SPPs wave formed through the holes in this direction due to destructive interference in this case. There is no existence of SPPs interfenence-based focusing accordingly. But an ideal circular focusing spot can be formed when we rotate the polarization direction to be $90^{\circ}$, i.e., the lens is under illumination with azimuthal polarization, as shown in Fig. 7 (b). It may be explained that the polarization direction is perpendicular to the long-axis of the elliptical pinholes, and constructive SPPs enhanced transmission and interference are generated accordingly. For more information regarding the influence of polarization states on focusing performance, readers can see our previous presentation in Ref. [25].

Finally, we addressed an additional issue here from FIB fabrication point of view. Real part of Au permittivity $\varepsilon_{\mathrm{Au}}$ will be increased due to $\mathrm{Ga}^{+}$implantation occurred during the FIB directly etching process [26]. It will cause variation of optical transmission property and focusing performance of the lens accordingly. Further study is necessary to reveal physical picture of the influence. 
Figure 7. (a) Calculated E-field intensity distribution $\left|E_{x}\right|^{2}$ at $x-y$ plane, $Z=0.6 \mu \mathrm{m}$ for (a) radial polarization and (b) azimuthal polarization.

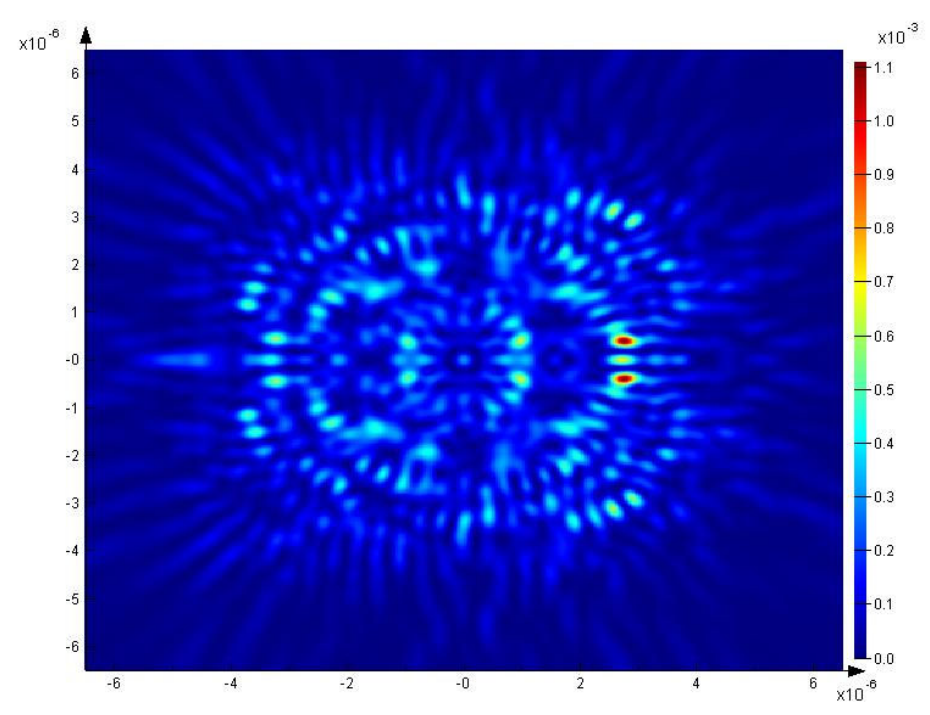

(a)

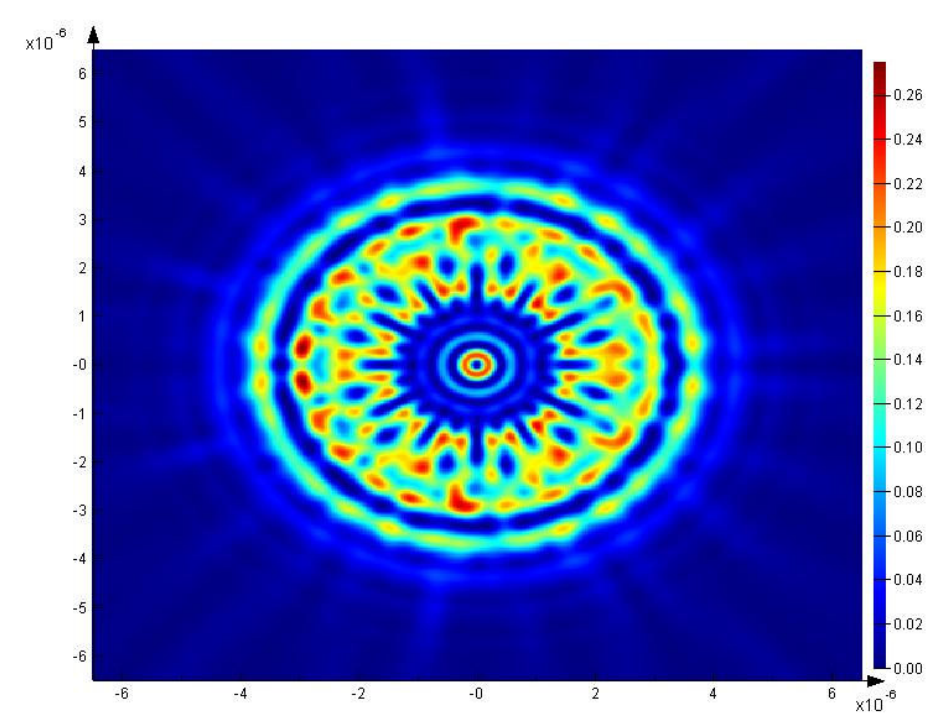

(b)

\section{Conclusions}

Transmission property and focusing performance of an elliptical nano-pinholes-based plasmonic lens were analyzed by means of both theoretical calculation and experimental nanofabrication and NSOM characterization. Optimized transmission property is derived at a ratio of short-axis to long-axis of the ellipses, $\delta=0.2$. The corresponding experimental results demonstrate that it is in agreement with the theoretical results. The lenses can realize subwavelength focusing with enhanced transmission, elongated focal length, and depth of focus which are crucial issues for the relevant applications such as nano-photolithography, superlensing, biosensing, and data storage. 


\section{Acknowledgements}

The work was supported by the National Natural Science Foundation of China (No.60877021 and 61077010).

\section{References and Notes}

1. Mark I. Stockman, Nanofocusing of Optical Energy in Tapered PlasmonicWaveguides, Phys. Rev. Lett., 93 (2004), 137404.

2. F. I. Baida - A. Belkhir, Superfocusing and light confinement by surface plasmon excitation through radially polarized beam. Plasmonics, 4 (2009), 51-59.

3. Yongqi Fu, W. Zhou, L. E. N. Lim, C. L. Du and X. G. Luo, "Plasmonic Microzone Plate: Superfocusing at visible regime”, Appl. Phys. Lett., 91(2007), 061124.

4. Yongqi Fu, Yu Liu, Xiuli Zhou, Shaoli Zhu, Experimental demonstration of focusing and lasing of plasmonic lens with chirped circular slits, Opt. Express, 18 (2010), 3438-3443.

5. Baohua Jia, Haofei Shi, Jiafang Li, Yongqi Fu, Chunlei Du, and Min Gu, Near-field visualization of focal depth modulation by step corrugated plasmonic slits, Appl. Phys. Lett., 94 (2009), 151912.

6. Yongqi $\mathrm{Fu}$, Xiuli Zhou, Yu Liu, Ultra-enhanced lasing effect of plasmonic lens structured with elliptical nano-pinholes distributed in variant period. Plasmonics, 5 (2010), 111-116.

7. Yongqi Fu, Xiuli Zhou, Topical review: plasmonic lenses, Plasmonics, 5 (2010). DOI 10.1007/s11468-010-9144-9.

8. Avner Yanai and Uriel Levy, Plasmonic focusing with a coaxial structure illuminated by radially polarized light, Opt. Express 17 (2009), 924-932.

9. Zhaowei Liu, Jennifer M. Steele, Werayut Srituravanich, Yuri Pikus, Cheng Sun, and Xiang Zhang, Focusing Surface Plasmons with a Plasmonic Lens, Nano Lett., 5 (2005), 1726-1729.

10. Zhaowei Liu, Jennifer M. Steele, Hyesog Lee, and Xiang Zhang, Tuning the focus of a plasmonic lens by the incident angle, Appl. Phys. Lett. 88 (2006), 171108.

11. Jennifer M. Steele, Zhaowei Liu, Yuan Wang and Xiang Zhang, Resonant and nonresonant generation and focusing of surface plasmons with circular gratings, Opt. Express. 14 (2006), 5664-5670.

12. Yongqi $\mathrm{Fu}$, Wei Zhou, Lim Enk Ng Lennie, Nano-pinhole-based optical superlens, Research Letter in Physics, Vol.2008 (2008), 148505.

13. Zhou Xiuli, Yongqi Fu, Wang Shi-Yong, Peng An-Jing, Cai Zhong-Heng, Funnelshaped arrays of metal nano-cylinders for nano-focusing, Chinese Phys. Lett.. 25 (2008), 3296-3299.

14. Gilad M. Lerman, Avner Yanai, and Uriel Levy, Demonstration of Nanofocusing by the use of Plasmonic Lens Illuminated with Radially Polarized Light, Nano Lett., 9 (2009), 2139-2143.

15. Qiwen Zhan, Advances in Optics and Photonics, 1 (2009), 1-57. 
16. L. Kipp, M. Skibowski, R. L. Johnson, R. Berndt, R. Adelung, S. Harm, and R. Seemann, Sharper images by focusing soft X-rays with photon sieves, Nature 414, 184-188 (2001).

17. R. Gordon, A.G. Brolo, Strong polarization in the optical transmission through elliptical nanohole arrays. Phys. Rev. Lett., 92, 037401 (2004).

18. R. Gordon and M. Hughes, B. Leathem and K. L. Kavanagh, A. G. Brolo, Basis and Lattice Polarization Mechanisms for Light Transmission through Nanohole Arrays in a Metal Film, Nano Lett., 5, 1243-1246 (2005).

19. Yongqi Fu, Wei Zhou, Lim Enk Ng Lennie, Chunlei Du, Influences of V-shaped plasmonic nanostructures on transmission properties. Appl. Phys. B, 86 (2007), 461-466.

20. R. Gordon, M. Hughes, B. Leathem and K. L. Kavanagh, and A. G. Brolo, Nano Lett., 5, 1243-1246, (2005).

21. Enoch, S.; Popov, E.; Neviere, M.; Reinisch, R. J. Opt. A: Pure Appl. Opt. 4, s83-s87 (2002).

22. Gilad M. Lerman, Avner Yanai, and Uriel Levy, Demonstration of nanofocusing by the use of plasmonic lens illuminated with radially polarized light. Nano Letters Vol.9, No.5, 2139-2143(2009)

23. F. Eftekhari, R. Gordon, J. Ferreira, A. G. Brolo, and D. Sinton, "Polarization-dependent sensing of a self-assembled monolayer using biaxial nanohole arrays", Appl. Phys. Lett. 92 (2008), 253103.

24. Yongqi Fu, Ngoi Kok Ann Bryan, Ong Nan Shing, Fabrication and characterization of nanopore array. J. Nanosci. Nanotechnol., 6, 1954-1960 (2006).

25. Zhenkui Shi, Yongqi Fu, Xiuli Zhou, Shaoli Zhu, Polarization effect on focusing of a plasmonic lens structured with radialized and chirped elliptical nanopinholes. Plasmonics, 5 (2010), 175-182.

26. Yongqi Fu, Ngoi Kok Ann Bryan, Investigation of physical properties of quartz via focused ion beam Bombardment. Appl. Phys. B, 80 (2005), 581-585.

(C) 2011 by the Authors; License Insciences Journal.

Open Access article under the terms and conditions of the Creative Commons Attribution Non-Commercial License 3.0 Unported 